\title{
Investigation of close interactions between sympathetic neural fibres and the follicular dendritic cells network in the mouse spleen
}

\author{
C. Demonceau, ${ }^{1}$ A.S. Marshall, ${ }^{2}$ J. Sales, ${ }^{3}$ E. Heinen ${ }^{1}$ \\ ${ }^{1}$ Institute of Human Histology, Immunology Centre, CRPP, University of Liège, Liège, Belgium; \\ ${ }^{2}$ Neuropathogenesis Unit, Institute for Animal Health, Ogston Building, Edinburgh, United Kingdom; \\ ${ }^{3}$ Biomathematics \& Statistics Scotland, Edinburgh, United Kingdom
}

(C)2008 European Journal of Histochemistry

In this study, co-localization between sympathetic neural fibres and the follicular dendritic cells (FDCs) network was observed within the mouse spleen by confocal technology. Immunohistochemical techniques were used to reveal the rare interactions between the FDCs network and sympathetic neural fibres. We estimated the frequency of three kinds of close interactions which could be defined as overlaps, contacts or neural fibres closer than $10 \mu \mathrm{m}$ from a FDCs network. Using these estimates, a comparison was made between five uninfected mouse strains exhibiting the same Prnp $^{a}$ genotype but showing different incubation periods when inoculated with primary bovine spongiform encephalopathy (BSE)-infected brain. In prion disease, infectivity is generally detected in the spleen much earlier than in the brain, especially after peripheral inoculation. The way by which the infectious agent reaches the central nervous system is still unclear. From the five mouse strains, we obtained differences in the proportion of splenic FDCs networks with close interactions. Our work suggests that the percentage of splenic FDCs networks with at least one sympathetic neural fibre in close vicinity may influence the length of incubation period.

Key words: Follicular dendritic cell, sympathetic nervous system, incubation period, prion.

Correspondence: Caroline Demonceau,

Institute of Human Histology, Immunology Centre,

CRPP, University of Liège, 1 Avenue de I'Hôpital,

4000 Liège, Belgium

Tel.: +32.4.366 5171 .

Fax: +32.4.366 4321 .

E-mail: cdemonceau@student.ulg.ac.be

Paper accepted on May 27, 2008

European Journal of Histochemistry

2008; vol. 52 issue 2 (Apr-Jun): 85-92
$\mathrm{T}$ ransmissible spongiform encephalopathies (TSEs), also called prion diseases, are fatal progressive neurodegenerative disorders of man and animals affecting the central nervous system (CNS). There is quite a large number of different prion strains, which can be distinguished in experimentally infected laboratory animals by particular durations of the asymptomatic incubation period and peculiar neuropathology profiles in inbred mouse strains (Bruce et al., 1991). For transmission of bovine spongiform encephalopathy (BSE) from cattle to mice, a replication phase in the spleen appears to be obligatory after peripheral infection before neuroinvasion can occur; this seems to be the case even when infection is introduced directly into the brain (Bruce et al., 1994). The intracerebral route is not faster than the intraperitoneal route and a peripheral processing step involving mature FDC may facilitate spread of BSE infection to the CNS (Brown et al., 1997).

Several lines of evidence indicate an involvement of the lymphoreticular system (LRS) in the development of prion diseases. After peripheral inoculation prions usually replicate in host's LRS tissues before neuroinvasion occurs (for review see Aguzzi \& Heikenwalder, 2006; Mabbott \& MacPherson, 2006). The key pathogenetic event in all animal and human TSEs is represented by the accumulation in the CNS, as well as in a number of peripheral nervous and LRS tissues, of a disease-specific PrP isoform ( $P r P d)$ of the normal host-encoded cellular prion protein $\left(\mathrm{Pr}^{\mathrm{c}}\right)$, with the incubation period and the clinico-pathological disease phenotype depending upon the host's PrP genotype and the prion strain (Farquhar et al., 1994; Farquhar et al., 1996). Pr $\mathrm{P}^{c}$ expression in peripheral tissues from both uninfected and infected mice is generally located on follicular dendritic cells (FDC) within lymphoid follicles, where it appears critical for prion replication and translocation to nerves (Kitamoto et al., 1991; McBride et al., 1992; O'Rourke et al., 
1994; Fraser et al., 1996; Klein et al., 1997; Klein et al., 1998; Brown et al., 1999; Mabbott et al., 2000; Raeber et al., 2001; Aguzzi \& Heikenwalder, 2006; Mabbott \& MacPherson, 2006). That FDC may be critical for prion infection pathogenesis in most species is also suggested by the presence of PrPd deposits in LRS tissues from patients with the variant Creutzfeldt-Jakob disease (vCJD; Hill et al., 1999) and from scrapie-affected sheep (van Keulen et al., 1996). However, FDC are non-migratory and how prion infection translocates into the peripheral and central nervous system is unknown (Mabbott and Bruce, 2003).

Evidence is accumulating on the role of the sympathetic nervous system (SNS) in neuroinvasion in several natural and experimental prion diseases. The sympathetic nodes of hamsters orally infected with scrapie accumulate PrPs (McBride and Beekes, 1999). Sympathectomy delays intraperitoneally routed scrapie in mice, and sympathetic hyper-innervation accelerated both replication within the spleen and neuroinvasion (Glatzel et al., 2001). Lymphoid organs are densely innervated by the SNS and there is evidence for direct interactions between noradrenergic fibres and lymphocytes and macrophages (Felten et al., 1985; Felten et al., 1987; Felten and Felten, 1988; Felten and Olschowka, 1987; Straub, 2004). Noradrenergic fibres have also been observed close to FDC in healthy sheep spleen, and to $\mathrm{Pr} \mathrm{P}^{\mathrm{sc}}$-accumulating cells in sheep affected by natural scrapie (Bencsik et al., 2001a; Bencsik et al., 2001b). A recent transgenic approach co-locating FDC with splenic nerves reduced the incubation period of intraperitoneally inoculated RML scrapie in mice, suggesting faster transfer into the central nervous system when sympathetic nerve endings are in the vicinity of FDC network (Prinz et al., 2003).

A non-transgenic model was used to investigate the sympathetic innervation of splenic FDC networks. As the incubation period is under the influence of the host Prnp genotype, the strains were chosen to have the same Prnpa gene allele to avoid any genetic effects of this gene on the incubation periods; Prnp, the gene that encodes for cellular prion protein, has two main variants in mice that differ only by their amino acids at coding positions 108 and 189 (Moore et al., 1998). Five mouse strains, that exhibit different incubation periods when inoculated with primary BSE, were included in the study. The three RIII-2, RIII-1 and 129/0la mouse strains have equivalent incubation periods which are 100-days shorter than those of C57BL and C57BL/6 (=B6) mouse strains: a mean incubation period of approximately 315 days compared to approximately 425 days for the C57BL strains. There is no evidence that these incubation periods have changed over the period since they were first reported (Bruce et al., 1994; Bruce et al., 1997; Fraser et al., 1992; Green et al., 2005; Manolakou et al., 2001; C. Farquhar, personal communication).

\section{Materials and Methods}

\section{Animals}

All five Prnpa mouse strains studied (RIII-1, RIII-2, 129/0la, C57BL, and C57BL/6 (subsequently referred to as $\mathrm{B} 6$ ) were bred under SPF conditions (except for RIII-1 which were maintained in quarantine), at the Neuropathogenesis Unit (NPU), Institute for Animal Health. For each mouse strain, 6 females ( 10 weeks old \pm 2 days) were sacrificed by cervical disruption and the spleen dissected. RIII-1 and RIII-2 are two different importations of the same mouse strain, but kept in different conditions due to welfare provisions. All protocols involving experimental animals were submitted to the Local Ethical Review Committee for approval and were performed under licence in accordance with the UK Home Office Regulations (Scientific Procedures) Act 1986.

\section{Immunohistochemistry}

Spleens were immersed in freshly prepared PLP (periodate-lysine-paraformaldehyde) fixative (0.0375 M phosphate buffer pH 7.4, 2\% paraformaldehyde, $0.1 \mathrm{M}$ sodium m-periodate, $0.075 \mathrm{M} \mathrm{D}$ L-lysine monohydrochloride) for six hours. Longitudinal slices of $100 \mu \mathrm{m}$ thickness were sliced with a vibratome (Oxford Vibratome) and kept in $0.05 \mathrm{M}$ Sörensen phosphate buffer, $\mathrm{pH} 7.4$ at $4^{\circ} \mathrm{C}$ into wells of a labelled 24-well plate until the next day.

For each spleen from each mouse, double-staining was performed upon three thick $100 \mu \mathrm{m}$ spleen slices: the $8^{\text {th }}, 11^{\text {th }}$ and $14^{\text {th }}$ sections from the concave side are representative of the whole mouse spleen. In general, sections 8 and 14 were at the extremities of the spleen with section 11 placed centrally within the spleen; sections 1 to 7 were extremely small 
and narrow. The total number of sections per spleen was between 15 and 16 sections with the last one sticking to the vibratome support. Immunohistochemistry was performed at room temperature and all solutions were prepared with Tris buffered saline $(7.45 \mathrm{~g}$ Tris $\mathrm{pH} 7.6,2 \mathrm{~g} \mathrm{BSA}, 8.766 \mathrm{~g} \mathrm{NaCl}$ in 1 I distilled water). After blocking for 20 min with normal goat serum (1:20, Dako), floating sections were incubated overnight with a polyclonal antityrosine hydroxylase antibody ( $\mathrm{TH}, 1: 200$, Chemicon) to identify sympathetic neural fibres, before washing, and incubating with a secondary detection anti-rabbit Alexa488 labelled antibody ( $15 \mu \mathrm{g} / \mathrm{mL}, B D$ Pharmingen) for one hour. To detect FDC networks, sections were incubated with monoclonal FDC-M2 biotinylated antibody (1:400, ImmunoKontact) for two hours. The sections were rinsed and incubated with streptavidin Alexa594 ( $2.5 \mu \mathrm{m} / \mathrm{mL}, B D$ Pharmingen) for one hour. Rinsed floating sections were delicately transferred onto slides and mounted with mounting fluorescent medium (Dako). Slides were then left to dry at $4^{\circ} \mathrm{C}$ overnight, coverslipped and sealed with nail varnish.

\section{Confocal analysis and morphometric measurements}

Analysis was performed upon vibratome-sectioned spleen tissue using a Zeiss LSM 5 Pascal confocal attached to a Zeiss Axioskop 2 MOT fluorescent microscope. All spleen sections were observed blind by only one investigator and the laser was retained at the same settings during analysis performed in this study. The z-stack series were recorded at $\times 63$ magnification lens with a 0.5 $\mu \mathrm{m}$ gap between each confocal scan, and the screen resolution determined that one pixel corresponded to $0.285 \mu \mathrm{m}$. Only the two external $10 \mu \mathrm{m}$ z-stack series scans, from each $100 \mu \mathrm{m}$-thick spleen section were analysed, as the FDC-M2 antibody used did not label throughout the section. In each confocal scan of the z-stack series, the counts of three kinds of close interactions between one tyrosine hydroxylase positive $\left(\mathrm{TH}^{+}\right)$neural fibres labelling and one individual extension from FDC network labelling were performed: the number of overlaps (pixels of both colours superimposed and thus appear as a yellow colour), contacts (pixels of both red and green colours adjacent to each other) and $\mathrm{TH}^{+}$neural fibres closer than $10 \mu \mathrm{m}$ from FDC network staining were counted for each spleen section. This was achieved by firstly observing the whole section at $\times 40$ magnification and the FDC network and TH staining pattern noted. In areas where FDC network and $\mathrm{TH}$ labelled cells were in proximity the section was then scanned with the $\times 63$ magnification lens. The Pascal software was used to measure distances and analysed colour superposition to confirm the close interactions observed. Morphometric measurements of area were performed with the Image$\mathrm{Pro}^{\circledast}$ PLUS software attached to a Zeiss fluorescent microscope.

For each of the three thick sections of the spleen per mouse, measurements were made of: the spleen area, the area of FDC network (as measured in the first confocal scan of the z-stack series from each side of the $100 \mu \mathrm{m}$ thick section), the number of FDC networks, and the number of neural fibres in close interactions with each FDC network for each confocal scan of the $10 \mu \mathrm{m}$ z-stack series. The number of overlaps between FDC network and neural fibres, the number of contacts between FDC network and neural fibres, and the number of neural fibres closer than $10 \mu \mathrm{m}$ from the FDC network were recorded separately. For each spleen section analysed, the spleen volume and the FDC networks volume analysed were estimated by multiplying respectively the area of the spleen section and the area of FDC networks inside this same spleen section by $10 \mu \mathrm{m}$ thickness of the z-stack series.

\section{Statistical methods}

The number of interactions between FDC networks and neural fibres was modelled using a generalised linear mixed model (GLMM) with a Poisson error and log link function. Individual analyses for each type of close interaction (overlaps, actual contacts or neural fibres closer than 10 $\mu \mathrm{m}$ from FDC network) and an overall analysis of the total number of close interactions were performed. The models fitted for each count had mouse strain, section and strain $x$ section interaction effects as fixed effects and the mice within strain as random effects. The dispersion parameter was estimated in the model as it would be expected that the method of counting would result in the data being over-dispersed. The proportion of FDC networks with neural fibres was modelled in the same way as the number of interactions except that a binomial distribution and logit link function were used in the GLMM. The percentage of spleen volume occupied by FDC networks was analysed using a general linear mixed model. Although differences in strains were observed for several of the variables of inter- 


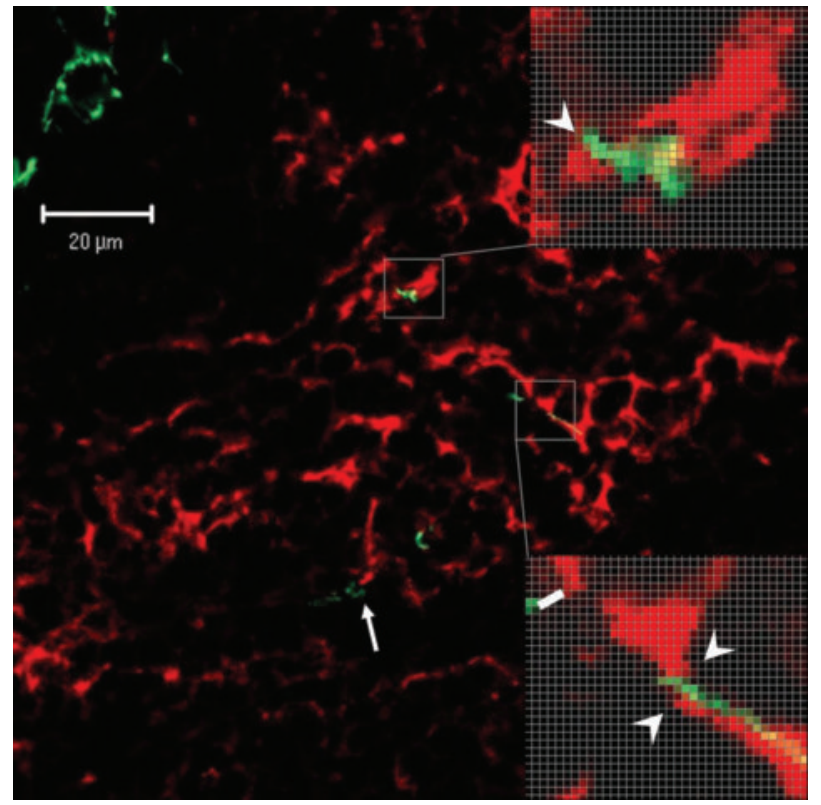

Figure 1. Confocal laser scanning microscopy of double immunofluorescent labelling with TH (green) and FDC-M2 (red) antibodies. We observed one confocal scan from a z-stack series showing the three types of close interactions: overlap (yellow colour from co-localization of green and red colours), contact between $\mathrm{TH}+$ neural fibres and FDC network (red and green colours side by side), and TH+ neural fibres closer than $10 \mu \mathrm{m}$ from FDC network. In the top left we observed a tubular-like structure associated with $\mathrm{TH}+$ labelling. The top right pixelbox shows a selected area where both yellow co-localization on the right and contact (arrowhead) with another FDC network labelling on the left are enlarged. The bottom right pixelbox shows a selected area where there are one yellow co-localization in the bottom right, two contacts (arrowheads) with different FDC network labellings in the centre, and one TH+ neural fibre closer than $\mathbf{1 0}$ $\mu \mathrm{m}$ from FDC network in the top left (large white bar represents $0.86 \mu \mathrm{m})$. The arrow points to a $\mathrm{TH}+$ neural fibre surrounding the FDC network.

est there did not appear to be any appreciable effect of section. Also, a similar pattern of results was obtained by looking at each type of close interactions separately or by looking at the total number of close interactions, consequently only results for total close interactions are reported; these are expressed as strain means with associated standard errors estimated from the simplified statistical models. Analyses were carried out using Genstat $8^{\text {th }}$ Edition (VSN International Ltd).

\section{Results}

Confocal analysis of spleen specimens examined revealed close interactions between sympathetic neural fibres and FDC network in all of them. Most of $\mathrm{TH}+$ neural fibres appeared to be associated with

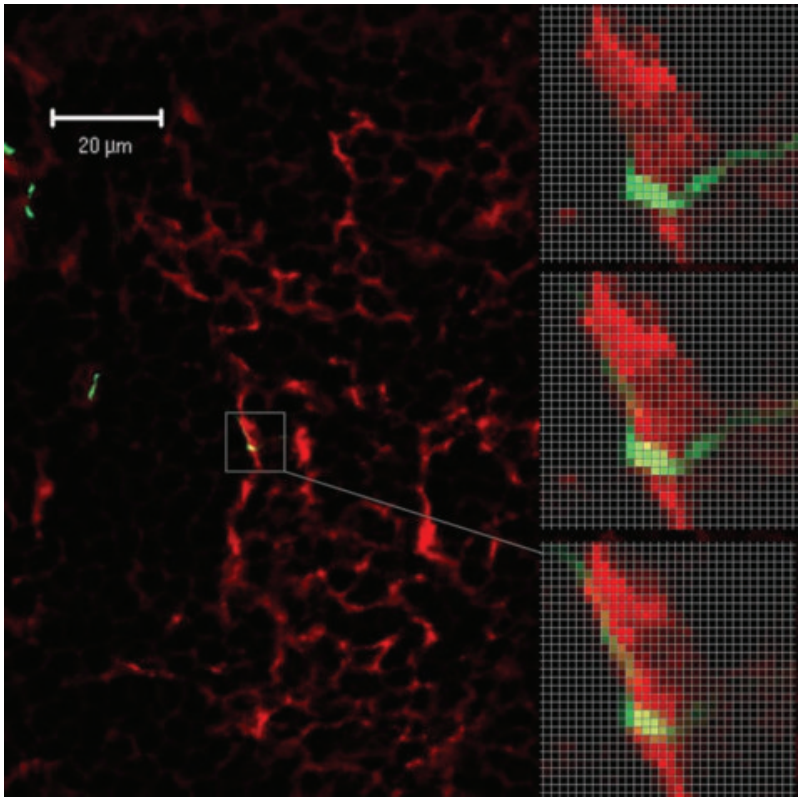

Figure 2. Confocal laser scan of double immunofluorescent labelling with TH (green) and FDC-M2 (red) antibodies. We observed one confocal scan from a z-stack series showing an overlap (yellow) between TH+ neural fibres (green) and FDC network (red). The bottom right pixelbox shows the selected area where yellow co-localization is enlarged. The upper pixelboxes are the two preceding serial confocal scans of the same area showing co-localization and contact respectively. Gap between confocal scan $=0.5 \mu \mathrm{m}$.

structures of tubular morphology with some projections going to the FDC network. Close interactions occasionally occurred at this interface and sometimes some neural fibres surrounded the FDC network (Figure 1). Figure 2 shows overlap between $\mathrm{TH}+$ neural fibres and FDC network (yellow pixels from co-localization of green- $\mathrm{TH}^{+}$and red-FDC network labels of the confocal scan). The bottom right pixelbox (Figure 2) represents a zoom of this overlap and the upper pixelboxes are the two preceding serial confocal scans of the z-stack series. It shows how a contact (pixels of both colours adjacent to each other) can move through a z-stack series to become an overlap at the second and third confocal scans 0.5 and $1 \mu \mathrm{m}$ away.

\section{The number and the volume of FDC networks}

Both within and between strains, there was a strong positive linear relationship between the number of FDC networks per unit volume of spleen and the percentage of spleen volume occupied by FDC networks (data not shown). Three mouse strains, RIII-2, RIII-1 and B6, showed the highest number of FDC networks $\left(2.4,2.2\right.$ and $2.4 / 10^{7} \mu \mathrm{m}^{3}$ spleen 
respectively) and the highest percentage of volume occupied by FDC network (5\%, 5.4\% and 3.4\% respectively). The lowest values were observed for the 129/0la mouse strain with 1.0 FDC networks $/ 10^{7} \mu^{3}$ spleen and $1.9 \%$ FDC network, and the C57BL mouse strain having intermediary values with 1.8 and $2.8 \%$ respectively (Table 1 ).

\section{The number of close interactions}

To estimate how much the FDC networks were sympathetically innervated between the five mouse strains, three kinds of close interactions were counted through the confocal scans of each z-stack series: overlaps, contacts and $\mathrm{TH}^{+}$neural fibres located closer than $10 \mu \mathrm{m}$ from a FDC network which are all represented in Figures 1 and 2 .

There were differences in the mean number of close interactions between the different strains. With reference to FDC network per unit volume, RIII-2 and 129/0la mouse strains showed the highest number of close interactions with $31.1 / 10^{7} \mu^{3}$ FDC network and 32.0/10 $\mu^{3}$ FDC networks respectively; the three RIII-1, C57BL and B6 mouse strains having a lower number of close interactions with 13.6, 17.8 and $18.7 / 10^{7} \mu^{3}$ FDC network respectively (Table 1 ). However, the mean number of close interactions, in FDC networks that had at least one close interaction, was very similar for all the five mouse strains and averaged about 4 close interactions per FDC network with close interactions (Table 1 ).

\section{The proportion of FDC networks with close inter- actions}

There was strong evidence that the proportion of FDC network that had at least one close interaction between $\mathrm{TH}^{+}$neural fibres and FDC network differed between strains $(p<0.001)$. The estimated percentages from the statistical fitted model are shown in Table 1. The RIII-2 strain had the highest estimated proportion with approximately $15 \%$ of FDC networks with close interactions between sympathetic neural fibres and FDC network whereas the C57BL and B6 strains had only 6\%; RIII-1 and 129/0la mouse strains had intermediary percentages with about $9 \%$ and $12 \%$ respectively (Table 1).

\section{Discussion}

In this study, the focus was on close interactions between sympathetic neural fibres and FDC networks in mouse spleen. An immunohistochemical approach was performed by means of confocal technology to have an overview of this neuroimmune interface within the spleen of five mouse strains which show different incubation periods when inoculated with primary BSE. No mouse strain which exhibits increased sympathetic innervation, for example KI4NGF transgenic mouse strain, was included in this study as the incubation period after inoculation with primary BSE is unknown.

There appeared to be no association between prion incubation periods in the different mouse strains and the density of FDC networks, or the percentage of splenic volume occupied by FDC networks (analyses involving the number of FDC networks or the volume of FDC networks gave similar informations). Three mouse strains, RIII-2, RIII-1 and $\mathrm{C} 57 \mathrm{BL} / 6$, have approximately 2.4 FDC networks $/ 10^{7} \mu \mathrm{m}^{3}$ spleen; the two first strains exhibit a short incubation period whereas the $\mathrm{C} 57 \mathrm{BL} / 6$ has a long incubation period. These results suggest that the FDC network hypertrophy is not a pre-required

Table 1. Means and (standard errors) estimated from fitting generalised linear mixed models to the data. RIII-2, RIII-1, 129/Ola mouse strains exhibit short incubation periods when infected with primary BSE; C57BL and B6 show long incubation periods after primary BSE infection.

\begin{tabular}{|c|c|c|c|c|c|}
\hline Mouse strain & RIII-2 & RIII-1 & 129/0la & C57BL & B6 \\
\hline Number of FDC networks per $10^{7} \mu \mathrm{m}^{3}$ spleen volume & $2.40(0.29)$ & $2.18(0.29)$ & $1.03(0.14)$ & $1.75(0.23)$ & $2.38(0.28)$ \\
\hline Volume of FDC network as a percentage of spleen volume & $5.00(0.38)$ & $5.37(0.41)$ & $1.86(0.39)$ & $2.77(0.39)$ & $3.39(0.38)$ \\
\hline Number of close interactions per $10^{7} \mu \mathrm{m}^{3}$ volume of FDC network & $31.1(4.0)$ & $13.6(2.6)$ & $32.0(7.0)$ & $17.8(4.0)$ & $18.7(2.8)$ \\
\hline Number of close interactions per number of FDC networks with close interactions & $4.23(0.53)$ & $3.74(0.61)$ & $4.18(0.73)$ & $4.25(0.75)$ & $4.26(0.58)$ \\
\hline Percentage of FDC networks with close interactions & $15.4(1.9)$ & $9.0(1.5)$ & $11.6(2.2)$ & $6.4(1.3)$ & $6.2(0.9)$ \\
\hline
\end{tabular}


for a more effective neuroinvasion. This observation corresponds with that of Prinz and co-workers' where, before or after RML prion strain infection, no relationship between FDC network hypertrophy and a shorter incubation period was observed (Prinz et al., 2003). Nonetheless, as hypertrophy of FDC dendrites was previously observed in C57BL mice infected with ME7 prion strain (McGovern et al., 2004), a similar phenomenon could be also induced during primary BSE infection in these five mouse strains, thus putatively exerting an effect upon neuroinvasion.

In regard to innervation, from all splenic lymphoid follicles examined in the five mouse strains, we generally observed sympathetic neural fibres associated with structures of tubular morphology, certainly the central artery, with thin neural fibres radiating away as described previously (Felten et al., 1985). Some neural fibres were also observed surrounding the FDC networks in lymphoid follicles, probably located at the mantle zone. For the first time, it was demonstrated that co-localization does occur between FDC networks and sympathetic neural fibres in the mouse spleen. This finding is in contrast to a previous study by our group that demonstrated that germinal centres of $\mathrm{C} 57 \mathrm{BL} / 6$ mouse strain, which contained FDC networks, are not innervated in Peyer's patches, another peripheral lymphoid tissue (Defaweux et al., 2005). However, our finding is supported by the work of Heggeb $\varnothing$ et al. in which Peyer's patches lymphoid follicles from Suffolk sheep were shown to harbour a more or less prominent nervous fibres (PGP9.5 $5^{+}$network, having close interactions with both FDC and so-called tingible body macrophages (Heggebø et al., 2003). Electronic microscopy and immunohistochemical studies would need to be combined to identify what kind of junction occurs between nerve endings and a dendrite characterized as coming from a FDC network.

In order to estimate FDC networks sympathetic innervation within the spleen of the five mouse strains, three kinds of close interactions between sympathetic neural fibres and FDC networks were counted in each scan of the $10 \mu \mathrm{m}$ z-stack series: overlaps (yellow confocal pixels from superposition of both red and green colours), contacts (confocal pixels from both red and green colours side by side) and $\mathrm{TH}^{+}$neural fibres closer than $10 \mu \mathrm{m}$ from FDC network. The method used gives evidence of how the $\mathrm{TH}^{+}$neural fibres interact with FDC networks in the volume of the spleen. Of particular interest was whether the number of these close interactions differed between the five mouse strains and whether any observed difference was related to the known differences in incubation period when inoculated with primary BSE.

The counts of close interactions gave information about the nature of the interface between sympathetic neural fibres and FDC networks. The RIII-2 and 129/0la mouse strains showed the highest number of close interactions per volume of FDC network, followed by the B6, C57BL and RIII-1 mouse strains.

For the five mouse strains, the mean number of close interactions for FDC networks with any close interactions appeared similar and averaged around four per network. Close interactions between TH neural fibres and FDC networks seemed to cluster within a few FDC networks as the same nerve could be reported several times throughout the confocal z-stack series. A 3D-structure would be needed to observe precisely where contacts occurred and to quantify the interface area between FDC and sympathetic neural fibres.

Of particularly interest was the number of FDC networks that had at least one close interaction compared to the total number of FDC network. The findings indicate that this proportion varies between mouse strains. The three mouse strains (RIII-2, RIII-1 and 129/0la) that have the highest proportions are also those that exhibit a shorter incubation period when inoculated with primary BSE. Rather than the actual number of neural fibres in close proximity to FDC network as proposed by Prinz et al. (2003), our work suggests that the percentage of FDC network with close interactions between sympathetic neural fibres and the FDC network may increase the possibility of neuroinvasion and thus cause a shorter incubation period in specific mouse strains.

With regard to prion diseases, the FDC seem to be implied in infectious agent replication before neuroinvasion by the sympathetic nervous system. Even the putative role of different $\operatorname{Pr}^{\mathrm{C}}$ expression levels are supposed, as well as, the multiple quantitative trait loci linked to differences in the incubation period (Lloyd et al., 2001); the aim of this study is to gain a better understanding of the additional/complementary role of the sympathetic nerve fibres/FDC networks interface. Spleens of five mouse strains with the same Prnpa genotype, but 
exhibiting different incubation periods when inoculated with primary BSE, were studied. Neither the mean density nor the volume of FDC networks for the different strains appeared to be related to the incubation periods. As FDC do not migrate, they cannot account for the transport of infectivity from the lymphoreticular system to the peripheral nervous system. However, real co-localizations do occur between TH neural fibres and FDC networks, and the number of close interactions could play a role in the time of access of prion agent to the CNS, and hence affect the incubation periods. Moreover, even if any immunostain of $\mathrm{PrP}^{\mathrm{sc}}$ could lead to the idea of a translocation by other cells (unlikely with regard to data from Raymond and Mabbott, 2007), our observation is still relevant compared with the theory of FDC-reservoir of PrPsc before spreading to sympathetic neural fibres. There is some evidence from this study that the proportion of FDC networks with close interactions may influence the incubation period but further work is needed to investigate this possibility.

\section{Acknowledgements}

This work has been supported by PrPScNEUROpathways research project number QLG3CT-2002-81030. CD would like to thank C.F. Farquhar, K.L. Brown, D. Ritchie and M. Bruce at the NPU for help and guidance on this project and the NPU for providing the mouse strains. The authors would also like to thank D. Silvestre for his support and useful discussions during the preparation of the paper.

\section{References}

Aguzzi A, Heikenwalder M. Pathogenesis of prion diseases: current status and future outlook. Nat Rev Microbiol 2006;4:765-75.

Bencsik A, Lezmi S, Hunsmann G, Baron T. Close vicinity of PrP expressing cells (FDC) with noradrenergic fibers in healthy sheep spleen. Dev Immun 2001a;8:235-241.

Bencsik A, Lezmi S, Baron T. Autonomic nervous system innervation of lymphoid territories in spleen: a possible involvement of noradrenergic neurons for prion neuroinvasion in natural scrapie. J Neurovirol 2001b;7:447-53.

Brown KL, Stewart K, Bruce ME, Fraser H. Severely combined immunodeficient (SCID) mice resist infection with bovine spongiform encephalopathy. J Gen Virol 1997;78:2707-10.

Brown KL, Stewart K, Ritchie DL, Mabbott NA, Williams A, Fraser H et al. Scrapie replication in lymphoid tissues depends on prion protein-expressing follicular dendritic cells. Nat Med 1999;5:1308-12.

Bruce ME, McConnell I, Fraser H, Dickinson AG. The disease characteristics of different strains of scrapie in Sinc congenic mouse lines: implications for the nature of the agent and host control of pathogenesis. J Gen Virol 1991;72(Pt 3):595-603.

Bruce ME, Chree A, McConnell I, Foster J, Pearson G, Fraser H. Transmission of bovine spongiform encephalopathy and scrapie to mice: strain variation and the species barrier. Philos Trans $\mathrm{R}$ Soc London B Biol Sci 1994;343:405-11.

Bruce ME, Will RG, Ironside JW, McConnell I, Drummond D, Suttie A et al. Transmissions to mice indicate that 'new variant' CJD is caused by the BSE agent. Nature 1997;389:498-501.

Defaweux V, Dorban G, Demonceau C, Piret J, Jolois 0, Thellin C et al. Interfaces between dendritic cells, other immune cells, and nerve fibres in mouse Peyer's patches: potential sites for neuroinvasion in prion diseases. Microsc Res Tech 2005;1;66:1-9.

Farquhar CF, Dornan J, Somerville RA, Tunstall AM, Hope J. Effect of Sinc genotype, agent isolate and route of infection on the accumulation of protease-resistant PrP in non-central nervous system tissues during the development of murine scrapie. J Gen Virol 1994; 75(Pt 3):495-504.

Farquhar CF, Dornan J, Moore RC, Somerville RA, Tunstall AM, Hope $J$. Protease-resistant PrP deposition in brain and non-central nervous system tissues of a murine model of bovine spongiform encephalopathy. J Gen Virol 1996;77(Pt 8):1941-6.

Felten DL, Felten SY, Carlson SL, Olschowka JA, Livnat $\mathrm{S}$. Noradrenergic and peptidergic innervation of lymphoid tissue. J Immunol 1985;135 (2 suppl.):755s-765s.

Felten DL, Ackerman KD, Wiegand SJ, Felten SY I. Nerve fibers associate with lymphocytes and macrophages in specific compartments of the splenic white pulp. J Neurosci Res 1987; 18:28-36, 118-21.

Felten DL, Felten S.Y. Sympathetic noradrenergic innervation of immune organs. Brain Behav Immun 1988; 2:293-300.

Felten SY, Olschowka J. Noradrenergic sympathetic innervation of the spleen: II. Tyrosine hydroxylase ( $\mathrm{TH}$ )-positive nerve terminals form synaptic-like contacts on lymphocytes in the splenic white pulp. J Neurosci Res 1987;18:37-48.

Fraser $\mathrm{H}$, Bruce ME, Chree A, McConnell I, Wells GAH. Transmission of bovine spongiform encephalopathy and scrapie to mice. J Gen Virol 1992; 73:1891-7.

Fraser H, Brown KL, Stewart K, McConnell I, McBride P, Williams A Replication of scrapie in spleens of SCID mice follows reconstitution with wild-type mouse bone marrow. J Gen Virol 1996;77:1935-40.

Glatzel M, Heppner FL, Albers KM, Aguzzi A. Sympathetic innervation of lymphoreticular organs is rate limiting for prion neuroinvasion. Neuron 2001;31:25-34.

Green R, Horrocks C, Wilkinson A, Hawkins SAC, Ryder SJ. Primary isolation of the bovine spongiform encephalopathy agent in mice: agent definition based on a review of 150 transmissions. J Comp Path 2005;132:117-31.

Heggeb $\varnothing$ R, González L, Press CM, Gunnes G, Espenes A, Jeffrey M. Disease-associated PrP in the enteric nervous system of scrapieaffected Suffolk sheep. J Gen Virol 2003;84(Pt5):1327-38.

Hill AF, Butterworth RJ, Joiner S, Jackson G, Rossor M N, Thomas DJ et al. Investigation of variant Creutzfeldt-Jakob disease and other human prion diseases with tonsil biopsy samples. Lancet 1999; 353:183-9.

Kitamoto T, Muramoto T, Mohri S, Doh-Ura K, Tateishi J. Abnormal isoform of prion protein accumulates in follicular dendritic cells in mice with Creutzfeldt-Jakob disease. J Virol 1991;65:6292-5.

Klein MA, Frigg R, Flechsig E, Raeber AJ, Kalinke U, Bluethmann H et al. A crucial role for $B$ cells in neuroinvasive scrapie. Nature 1997;390:687-90.

Klein MA, Frigg R, Raeber AJ, Flechsig E, Hegyi I, Zinkernagel RM et al. PrP expression in $B$ lymphocytes is not required for prion neuroinvasion. Nat Med 1998;4:1429-33.

Lloyd SE, Onwuazor ON, Beck JA, Mallinson G, Farrall M, Targonski $P$, et al. Identification of multiple quantitative trait loci linked to prion disease incubation period in mice. Proc Natl Acad Sci 2001;98:6279-83.

Mabbott NA, Farquhar CF, Brown KL, Bruce ME. Involvement of the immune system in TSE pathogenesis. Immunol Today 1998; 19:201-3.

Mabbott NA, Mackay F, Minns F, Bruce ME. Tempororary inactivation of follicular dendritic cells delays neuroinvasion of scrapie. Nat Med 2000;6:719-20.

Mabbott NA, Bruce ME. Prion disease: bridging the spleen-nerve gap. Nat Med 2003;9:1463-4.

Mabbott NA, MacPherson GG. Prions and their lethal journey to the 


\section{Demonceau et al.}

brain. Nat Rev Microbiol 2006;4:201-11.

Manolakou K, Beaton J, McConnell I, Farquhar CF, Manson J, Hastie $N D$, et al. Genetic and environmental factors modify bovine spongiform encephalopathy incubation period in mice. Proc Natl Acad Sci USA 2001;98:7402-7.

McBride PA, Eikelenboom P, Kraal G, Fraser H, Bruce ME. PrP protein is associated with follicular dendritic cells of spleens and lymph nodes in uninfected and scrapie-infected mice. J Pathol 1992; 168:413-8.

McBride PA, Beekes M Pathological PrP is abundant in sympathetic and sensory ganglia of hamsters fed with scrapie. Neurosci Lett 1999; 265:135-8.

McGovern G, Brown KL, Bruce ME, Jeffrey M. Murine scrapie infection causes an abnormal germinal centre reaction in the spleen. J Comp Path 2004; 130:181-94.

Moore RC, Hope J, McBride PA, McConnell I, Selfridge J, Melton DW, et al. Mice with gene targetted prion protein alterations show that Prnp, Sinc and Prni are congruent. Nat Genet 1998; 18:118-25.
O'Rourke KI, Huff TP, Leathers CW, Robinson M M, Gorham JR. SCID mouse spleen does not support scrapie agent replication. J Gen Virol 1994;75:1511-4.

Prinz M, Heikenwalder M, Junt T, Schwarz P, Glatzel M, Heppner FL et al. Positioning of follicular dendritic cells within the spleen controls prion neuroinvasion. Nature 2003;425:957-62.

Raeber AJ, Montrasio F, Hegyi I, Frigg R, Klein MA, Aguzzi A et al. Studies on prion replication in spleen. Dev Immunol 2001;8:291304.

Raymond $\mathrm{CR}$, Mabbott NA. Assessing the involvement of migratory dendritic cells in the transfer of the scrapie agent from the immune to peripheral nervous systems. J Neuroimmunol 2007; 187:114-25.

Straub RH. Complexity of the bi-directional neuroimmune junction in the spleen. Trends Pharmacol Sci 2004;25:640-6.

van Keulen LJ, Schreuder BE, Meloen RH, Mooij-Harkes G, Vromans $M E$, Langeveld JP. Immunohistochemical detection of prion protein in lymphoid tissues of sheep with natural scrapie. J Clin Microbiol $1996 ; 34: 1228-31$ 